Rev. Biol. Trop. 52(1): 85-95, 2004

www.ucr.ac.cr www.ots.ac.cr www.ots.duke.edu

\title{
Ácaros oribátidos (Acari: Oribatei) del Parque Nacional de la isla de Coiba, Panamá
}

\author{
L. Subías ${ }^{1}$, J.P. Zaballos ${ }^{1}$, E. Banda ${ }^{1}$, F. Fontal-Cazalla² \& J.L. Nieves-Aldrey ${ }^{2}$ \\ 1 Departamento Biología Animal I. Facultad de Biología. Universidad Complutense de Madrid. 28040 Madrid. \\ 2 Museo Nacional de Ciencias Naturales (CSIC). José Gutiérrez Abascal 2. Madrid 28006.
}

\author{
Recibido 10-III-2001. C Corregido 03-VIII-2001. Aceptado 08-II-2002.
}

\begin{abstract}
Taking part of the Program for The Inventory of Flora and Fauna of Coiba Island National Park (Panamá), a first list of oribatid mites (Acari: Oribatei) is presented. Materials studied come from several soil samplings, including humus and fallen leaves, done in Coiba Island in July 1998. 134 species were identified from a total amount of 1053 individuals collected. Almost all the identified species are new records for Panamá as well as eigth species that represent first records for the Neotropical Region. A new name is given: Aeroppia mariehammerae n. nom. for Aeroppia sp. and a new combination is proposed: Protoribates antillensis (Mahunka, 1985) n. comb. (Xylobates).
\end{abstract}

Key words: Oribatid mites, Coiba island, Panamá, Neotropic.

La isla de Coiba, con una extensión aproximada de 50000 ha, es la más grande del Pacífico centroamericano. Situada a unos $25 \mathrm{~km}$ del continente, en la provincia de Veraguas, en el Sudoeste de la República de Panamá, desde 1991 forma parte principal del Parque Nacional de su mismo nombre, en el que también se integran ocho islas menores, treinta islotes y una extensa área marina, constituyendo uno de los espacios naturales mejor preservados de Panamá.

Gran parte de los ecosistemas y hábitats naturales de Coiba, entre los que destacan un $80 \%$ de bosques húmedos tropicales, se encuentran en excelente estado de conservación gracias al mantenimiento, desde hace más de 70 años, de una colonia penal en la isla que ha impedido su colonización y degradación.

Desde 1993, enmarcado en un convenio de cooperación hispano-panameño, equipos mixtos de investigadores de España y Panamá, coordinados por Santiago Castroviejo (Real Jardín Botánico, CSIC), vienen realizando tra- bajos de inventario de flora y fauna del Parque Nacional de Coiba; el primer fruto de este trabajo se publicó en 1997, en un libro colectivo que recoge los resultados preliminares del inventario de plantas vasculares y de muchos grupos zoológicos (Castroviejo 1997). Dada la ausencia de estudios previos, la mayor parte de dichos resultados eran los primeros datos publicados sobre la flora y la fauna de la citada área geográfica. Entre los grupos zoológicos estudiados en el mencionado volumen, se incluían ácaros de agua dulce (Hydrachnellae) (Camacho et al. 1997), pero no ácaros terrestres.

En una expedición entomológica al Parque Nacional, realizada durante dos semanas de julio de 1998, tuvimos ocasión de recoger distintas muestras de suelo, en varios puntos de la isla de Coiba, de las cuales se extrajeron gran cantidad de ácaros oribátidos. El resultado del estudio de este material se presenta aquí, representando los primeros datos de dicha fauna en el Parque Nacional. 
El conocimiento actual de los oribátidos, a nivel mundial, es muy deficiente; existen grandes regiones todavía inexploradas en este campo, y en las mejor estudiadas, como es el caso de Europa en donde hay amplias recopilaciones en Ghilarov y Knivolutsky (1975) y Subías y Gil-Martín (1997), aún siguen describiéndose nuevas especies.

En la región zoogeográfica neotropical, el estudio de los ácaros oribátidos es bastante tardío, destacando las descripciones de Antonio Berlese (1888) a principio de siglo y los trabajos, ya más profundos, de M. Hammer (1958, 1962) a mediados de siglo. No obstante, sin olvidar las aportaciones menores, cabe destacar los trabajos de J. Balogh y S. Mahunka, que culminan en una importante recopilación actualizada, con claves de identificación, en los dos volúmenes de Balogh y Balogh (1988, 1990) que incluyen cerca de 2000 especies de ácaros oribátidos. Este número de especies estaría muy por debajo de una estimación realista. Este es el caso de Panamá, ya que hasta la fecha sólo existían algunas citas aisladas. Se incluyen en este trabajo un listado de 134 especies de oribátidos de Panamá (de las que un buen número son posiblemente nuevas para la ciencia), lo que supone una importante contribución a los estudios de este tipo de fauna en la región neotropical.

\section{MATERIALES Y MÉTODOS}

Las muestras fueron recolectadas y manipuladas por J.P.Z. (salvo la número PC-11, que fue recogida por Alicia Ibáñez). Todas ellas proceden de la isla de Coiba en el Parque Nacional de Coiba (Panamá). Las muestras estudiadas fueron recogidas en el campo, tamizando enérgicamente una abundante cantidad de hojarasca, humus y los primeros centímetros de suelo. Se utilizó un tamizador con una luz de malla de $5 \mathrm{~mm}$ y la muestra obtenida se transportó, en el día, hasta el laboratorio de la Estación Biológica.

Las muestras tamizadas se colocaron en aparatos de Berlese portátiles (Coiffait 1958) con luz de malla de $2 \mathrm{~mm}$. La fauna obtenida se recogió en agua jabonosa y se conservó en líquido Scheerpeltz (60\% alcohol 97, $39 \%$ agua destilada, $1 \%$ ácido acético puro). Debido a la elevada humedad ambiental y a la falta de suministro eléctrico diurno, las muestras no se desecaban totalmente, por lo que se realizaron lavados con abundante agua dulce de las muestras tamizadas. El sobrenadante de cada muestra, se filtró con tela porosa y, una vez bien escurrido, se colocaron en los aparatos de Berlese portátiles. De esta forma se aceleró el sistema de secado, aunque esto supusiera la pérdida de parte de la información. Las muestras así obtenidas, fueron separadas y estudiadas en el Departamento Biología Animal I de la Universidad Complutense de Madrid.

\section{RESULTADOS}

Referencia y descripción de las muestras estudiadas: Se recolectaron y estudiaron un total de 16 muestras, que con la referencia PC-n, se describen a continuación. Los 9 puntos de muestreo seleccionados, con referencia a las muestras colectadas, se representan en la figura 1.

PC-1: Campamento Playa Blanca (17NMU1516, 20 m). 21de julio, 1998. Heliconia humus-2.

Entre alta vegetación herbácea se realizó un tamizado de humus bajo Heliconia. La muestra obtenida se colocó directamente en los aparatos de Berlese. En la muestra, además de los ácaros, se encontraron, arañas (3), opiliones (1), pseudoscorpiones (7), miriápodos, colémbolos (27), estafilínidos (Coleoptera) (12), carábidos (Coleoptera) (4), pseláfidos (Coleoptera) (7), otros coleópteros (18), larvas de insectos (4), dípteros (10), hemípteros (3), formícidos (Hymenoptera) (57), otros himenópteros (1) e isópodos (Crustacea) (14).

PC-2: La Falla (Playa Hermosa) (17NMU0531, 10 m). 26 de julio, 1998.

Se tamizó una abundante muestra de aluviones de río formado por hojarasca, fragmentos 


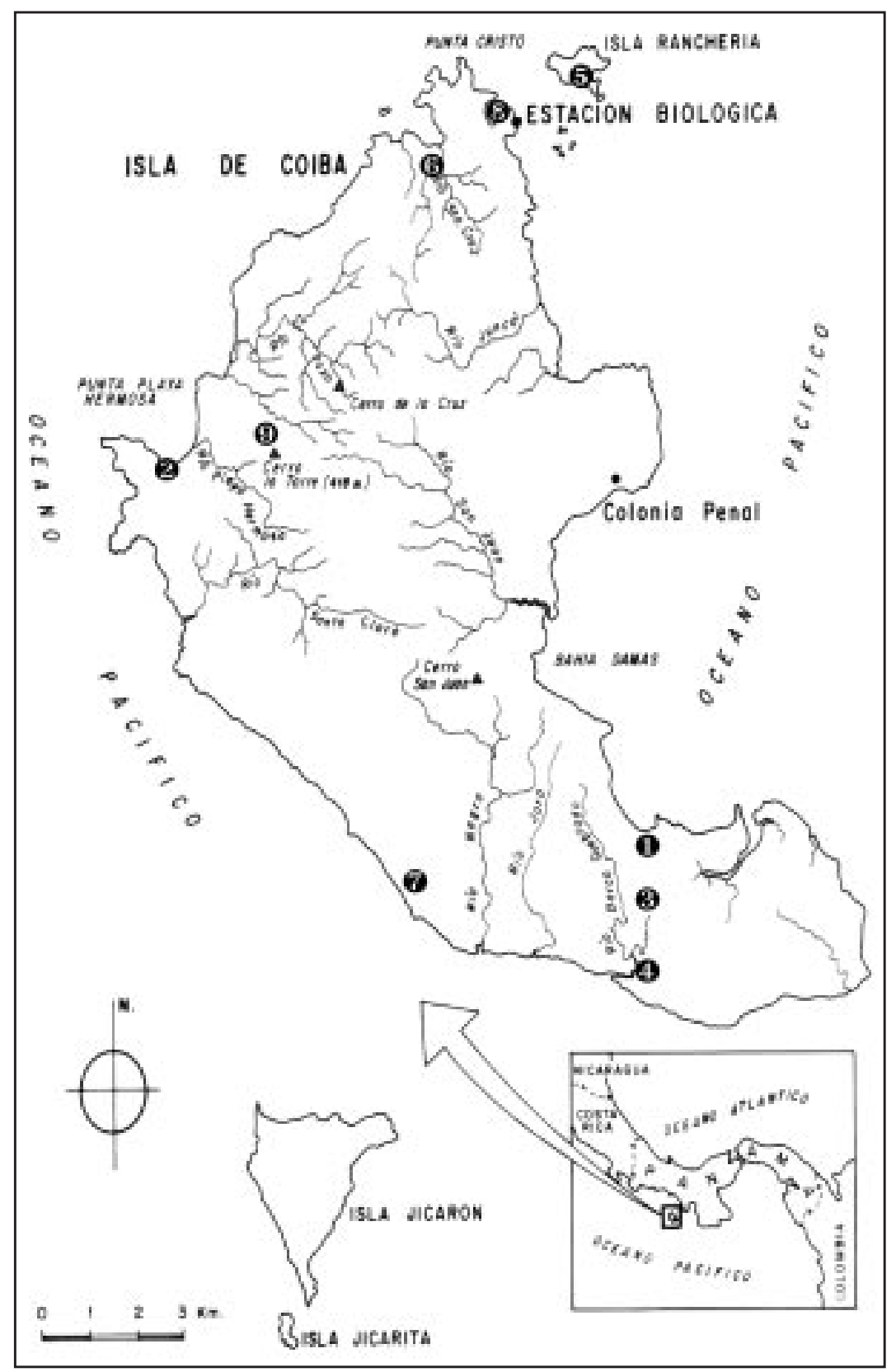

Fig. 1. Mapa del Parque Nacional de Coiba con la localización de los puntos de muestreo, indicando las referencias de las muestras recolectadas: 1. Campamento Playa Blanca (muestras PC-1, PC-5); 2. La Falla en Playa Hermosa (PC-2, PC-6); 3. Los Cativales (PC-3); 4. Barco Quebrado (PC-4); 5. Isla Ranchería (Coibita) (PC-7, PC-9, PC-12, PC-16); 6. Manglar de Santa Cruz (PC-10); 7. Campamento Manila (PC-11); 8. Estación Biológica y sendero de Alex (PC-13, PC-14); 9. Cerro de la Torre (PC-15).

Fig. 1. Map of The National Park of Coiba Island showing sampling sites and indicating the reference of each collected sample: 1. Campamento Playa Blanca (samples PC-1, PC-5); 2. La Falla en Playa Hermosa (PC-2, PC-6); 3. Los Cativales (PC3); 4. Barco Quebrado (PC-4); 5. Isla Ranchería (Coibita) (PC-7, PC-9, PC-12, PC-16); 6. Manglar de Santa Cruz (PC-10); 7. Campamento Manila (PC-11); 8. Estación Biológica and sendero de Alex (PC-13, PC-14); 9. Cerro de la Torre (PC-15). 
vegetales de tamaño diverso y arena. La muestra obtenida fue lavada y filtrada en el laboratorio y posteriormente colocada en los aparatos de Berlese. En la muestra, además de los ácaros, se encontraron, arañas (1), pseudoscorpiones (2), miriápodos (15), colémbolos (2), dipluros (2), estafilínidos (39), carábidos (4), escarabeidos (1), curculiónidos (1), otros coleópteros (34), larvas de insectos (15), dípteros (33), hemípteros (2), formícidos (97) y lepidópteros (2).

PC-3: Los Cativales (17NMU2615, 20 m). 21 de julio, 1998. En el bosque semi-inundado de cativo (Prioria copaifera) que se encuentra entre Playa Blanca y Barco Quebrado.

Aunque ha sido intervenido por el hombre, tiene un gran desarrollo con árboles de gran porte. Se realizó un tamizado de hojarasca y humus de cativo. La muestra obtenida se colocó directamente en los aparatos de Berlese. En la muestra, además de los ácaros, se encontraron, arañas (1), estafilínidos (3), pseláfidos (2), otros coleópteros (1), dípteros (1), tisanópteros (1) y formícidos (6).

PC-4: Barco Quebrado (17NMU2310, 10 m). 21 de julio, 1998.

En un cacaotal abandonado se realizó un tamizado de hojarasca y humus bajo un árbol de cacao. La muestra obtenida se colocó directamente en los aparatos de Berlese. En la muestra, además de los ácaros, se encontraron, arañas (2), pseudoscorpiones (2), miriápodos (3), estafilínidos (1), otros coleópteros (14), larvas de insectos (6), dípteros (1), hemípteros (2), formícidos (13) y otros himenópteros (1).

PC-5: Campamento Playa Blanca (17NMU1516, 20 m). 21 de julio, 1998. Heliconia humus-1.

Entre alta vegetación herbácea se realizó un tamizado de hojarasca bajo Heliconia, la muestra obtenida se colocó directamente en los aparatos de Berlese. En la muestra, además de los ácaros, se encontraron, pseudoscorpiones (2), colémbolos (2), dipluros (2), pseláfidos (3), otros coleópteros (1), formícidos (18) e isópodos (2).
PC-6: La Falla (Playa Hermosa) (17NMU0531, 80 m). 20 de julio, 1998.

Se tamizó una abundante cantidad de hojarasca y humus de Calophyllum (de nombre vernáculo María) junto con raíces de helechos de pequeño porte. La muestra obtenida se colocó directamente en los aparatos de Berlese. En la muestra, además de los ácaros, se encontraron, arañas (3), opiliones (2), pseudoscorpiones (9), miriápodos (25), colémbolos (3), estafilínidos (Coleoptera) (9), carábidos (Coleoptera) (11), pseláfidos (Coleoptera) (1), otros coléopteros (15), larvas de insectos (5), dípteros (47), hemípteros (26), formícidos (Hymenoptera) (78) y lepidópteros (3).

PC-7: Isla Coibita (Ranchería) (17NMU2244, 20 m). 25 de julio, 1998. Se tomó una muestra de tamizado de hojarasca y humus de Artocarpus communis (árbol del pan). La muestra obtenida fue lavada y filtrada en el laboratorio, y posteriormente colocada en los aparatos de Berlese. En la muestra, además de los ácaros, se encontraron, arañas (1), miriápodos (2), estafilínidos (8), carábidos (3), pseláfidos (2), otros coleópteros (2), larvas de insectos (3), dípteros (14), hemípteros (1), formícidos (10) y otros himenópteros (1).

PC-8: Isla Coibita (Ranchería) (17NMU2244, 10 m). 25 de julio, 1998.

Se tamizó una abundante muestra de hojarasca de Calophyllum y bambú (Bambusa) junto al camino que sube a la casa de la isla. La muestra obtenida fue lavada y filtrada en el laboratorio, y posteriormente colocada en los aparatos de Berlese. En la muestra, además de los ácaros, se encontraron, arañas (2), pseudoscorpiones (6), miriápodos (2), colémbolos (1), psocópteros (1), estafilínidos (5), carábidos (1), escarabeidos (1), otros coleópteros (8), dípteros (19) y formícidos (6).

PC-9: Isla Coibita (Ranchería) (17NMU2244, 10 m). 25 de julio, 1998.

Se tamizó una muestra de hojarasca de $\mathrm{Ca}$ lophyllum y cocotero (Cocos nuccifera) junto al camino. La muestra obtenida fue lavada y 
filtrada en el laboratorio, y posteriormente colocada en los aparatos de Berlese. En la muestra, además de los ácaros, se encontraron, arañas (1), psocópteros (1), estafilínidos (3), otros coleópteros (6), larvas de insectos (2), dípteros (16), formícidos (29) y otros himenópteros (1).

PC-10: Santa Cruz (17NMU1443, 0 m). 24 de julio, 1998.

Junto al manglar de la playa de Santa Cruz, se recogió una abundante muestra de tamizado de hojarasca de Calophyllum, humus y unos dos centímetros de suelo. La muestra obtenida fue lavada y filtrada en el laboratorio, y posteriormente colocada en los aparatos de Berlese. En la muestra, además de los ácaros, se encontraron, pseudoscorpiones (2), miriápodos (2), colémbolos (8), estafilínidos (7), carábidos (3), escarabeidos (1), pseláfidos (1), otros coleópteros (8), larvas de insectos (1), dípteros (8), tisanópteros (2), hemípteros (5), formícidos (15) y otros himenópteros (1).

PC-11: Campamento Manila (17NMU1413, 10 m). 23 de julio, 1998.

Muestra de humus lavada y filtrada en el laboratorio, y después colocada en los aparatos de Berlese. En la muestra, además de los ácaros, se encontraron, colémbolos (1), pseláfidos (1), larvas de insectos (1), dípteros (3), formícidos (5) e isópodos (1).

PC-12: Isla Coibita (Ranchería) (17NMU2244, 10 m). 19 de julio, 1998.

Muestra de tamizado de hojarasca y humus de Bambú (Bambusa) y cocotero (Cocos nuccifera), fue tomada al lado del camino que sube de la playa a la casa de la isla. La muestra obtenida se colocó directamente en los aparatos de Berlese. En la muestra, además de los ácaros, se encontraron, arañas (8), opiliones (3), pseudoscorpiones (2), miriápodos (9), colémbolos (1), estafilínidos (1), pseláfidos (1), otros coleópteros (1), larvas de insectos (1) y formícidos (10).
PC-13: Estación Biológica (17NMU1942, 0 m). 23 de julio, 1998.

Se realizó un lavado de tierra recolectada en la parte posterior del laboratorio de la estación biológica. Una vez filtrada se colocó en un aparato de Berlese. En la muestra, además de los ácaros, se encontraron, pseudoscorpiones (6), miriápodos (13), colémbolos (14), proturos (1), estafilínidos (2), carábidos (1), escarabeidos (3), otros coleópteros (4), larvas de insectos (5), dípteros (13), tisanópteros (3), hemípteros (1), formícidos (3) y dictiópteros (1).

PC-14: Estación Biológica (Sendero de Alex) (17NMU1842, 20 m). 22 de julio, 1998.

Muestra de tamizado de humus y suelo superficial en un bosque secundario con algunos Apeiba tibourbou (peine de mono) situado a unos $300 \mathrm{~m}$ de la estación biológica en dirección norte. Después de lavada y filtrada fue colocada en aparatos de Berlese. En la muestra, además de los ácaros, se encontraron, pseudoscorpiones (77), miriápodos (3), colémbolos (7), estafilínidos (1), pseláfidos (1), otros coleópteros (4), larvas de insectos (1), dípteros (1) y formícidos (48).

PC-15: Cerro de la Torre (17NMU0930, $300 \mathrm{~m}) .18-7-98$.

Se recogió una abundante muestra de tamizado de hojarasca, humus y primeros centímetros de suelo en bosque de Calophyllum. La muestra obtenida se colocó directamente en los aparatos de Berlese. En la muestra, además de los ácaros, se encontraron arañas (11), opiliones (3), pseudoscorpiones (10), miriápodos (5), colémbolos (3), psocópteros (1), estafilínidos (15), carábidos (2), pseláfidos (1), curculiónidos (1), otros coleópteros (7), larvas de insectos (6), dípteros (6), tisanópteros (2), hemípteros (6), formícidos (116), dictiópteros (2) e isópodos (1).

PC-16: Isla Coibita (Ranchería) (17NMU2244, 30 m). 19 de julio, 1998. 
Al final de la pista de aterrizaje de la isla, se tomó una muestra de tamizado de hojarasca y humus de Calophyllum. La muestra obtenida se colocó directamente en los aparatos de Berlese. En la muestra, además de los ácaros, se encontraron, arañas (2), opiliones (2), pseudoscorpiones (3), miriápodos (1), colémbolos (2), psocópteros (1), estafilínidos (1), pseláfidos (3), otros coleópteros (12), larvas de insectos (2), hemípteros (1), formícidos (43), isópodos (1).

\section{Listado sistemático de especies}

Para cada especie se señalan las referencias de las muestras en las que aparece, indicando entre paréntesis el número de ejemplares obtenido en cada una de ellas.

Las especies marcadas con el símbolo* se citan por primera vez para la región neotropical.

BRACHYCHTHONIIDAE

Sellnickochthonius sp. .

PC-1(1)

GEHYPOCHTHONIIDAE

*Gehypochthonius rhadamanthus Jacot, 1936

PC-2(1)

HYPOCHTHONIIDAE

Eohypochthonius gracilis (Jacot, 1936) ..........PC-1(15), PC-2(8), PC-4(5), PC-5(2), PC-10(2), PC-11(1), PC-14(1)

MESOPLOPHORIDAE

?Archoplophora sp. . . . . . . . . . . . . . . . . . . . . . . . . . . . . ..PC-1(1), PC-10(2)

Mesoplophora cubana Calugar y Vasiliu, 1977 . . . PC-1(1), PC-6(2), PC-10(4), PC-11(1), PC-12(2), PC-15(4), PC-16(2)

EPILOHMANNIIDAE

Epilohmannia pallida Wallwork, 1962 ..........PC-2(5), PC-7(1), PC-10(1), PC-11(1), PC-12(1), PC-13(1), PC-14(1)

Epilohmannia sp. . . . . . . . . . . . . . . . . . . . . . . . . . . . . . . . . .PC-2(2), PC-10(1)

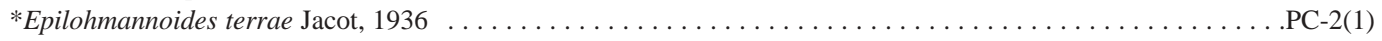

LOHMANNIIDAE

Annectacarus mucronatus Grandjean, $1950 \ldots \ldots \ldots \ldots \ldots \ldots \ldots \ldots \ldots \ldots \ldots \ldots \ldots$. . . 2 (181), PC-11(1), PC-12(1)

Javacarus porosus Hammer, 1979 . . . . . . . . . . . . . . . . . . . . . . . . . . . . . . . . PC-2(22), PC-11(1), PC-13(18)

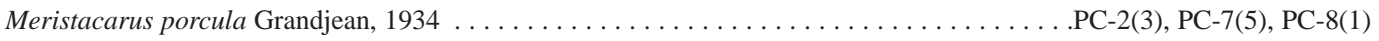

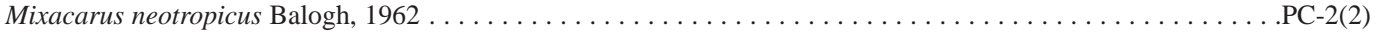

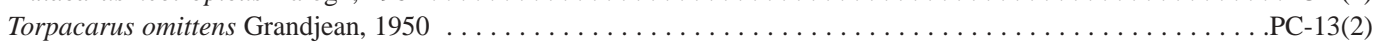

ORIBOTRITIIDAE

Indotritia krakatauensis (Sellnick, 1923) . . . . . . . PC-1(1), PC-4(1), PC-7(1), PC-11(1), PC-13(3), PC-14(10), PC-15(2)

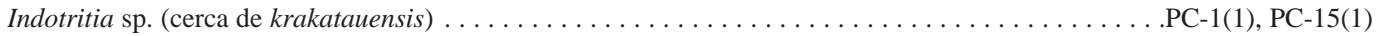

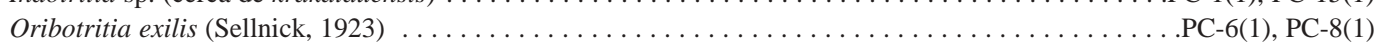

EUPHTHIRACARIDAE

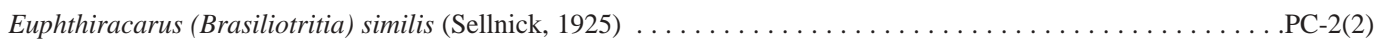

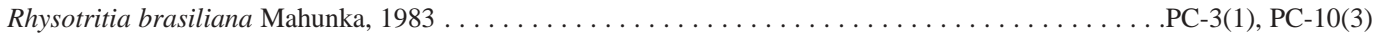

Rhysotritia clavata Märkel, $1964 \ldots \ldots \ldots \ldots \ldots \ldots \ldots \ldots \ldots \ldots \ldots \ldots \ldots \ldots \ldots \ldots \ldots$. . . . . . . . . . . . . . . . . . . PC-11(7)

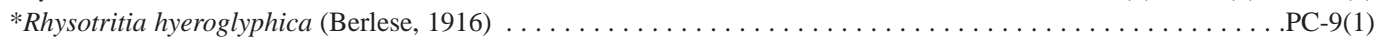

PHTHIRACARIDAE

Notophthiracarus (Austrophthiracarus) caudatus (Bal. y Mah., 1977) . . . . . . . . . . . . . . . .PC-2(1), PC-3(1)

Notophthiracarus (Austrophthiracarus) sp. (cerca de caudatus) . . . . . . . . . . . . . . . . . . . .PC-2(2)

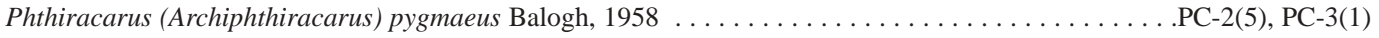

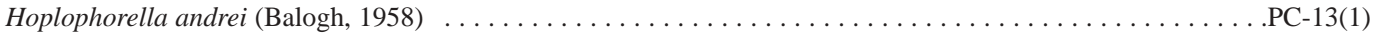

Hoplophorella cf. lanceosetoides Mahunka, $1985 \ldots \ldots \ldots \ldots \ldots \ldots \ldots \ldots \ldots \ldots \ldots \ldots \ldots \ldots \ldots \ldots$. . . . . . . . . . . . . . . . 
MALACONOTHRIDAE

Malaconothrus hauseri Mahunka, 1983

PC-3(1), PC-6(1), PC-7(1)

TRHYPOCHTHONIIDAE

Afronothrus incisivus Wallwork, $1961 \ldots \ldots \ldots \ldots \ldots \ldots \ldots \ldots \ldots \ldots \ldots \ldots \ldots$. . . . . . . . . . . 1), PC-10(3), PC-12(6)

Allonothrus neotropicus Balogh y Mahunka, $1969 \ldots \ldots \ldots \ldots \ldots \ldots \ldots \ldots \ldots \ldots \ldots \ldots$. . . . . . . . . . . . . . . . . . . . . . . . . . . . . . . . . . . . . . . . . . . PC-7(3)

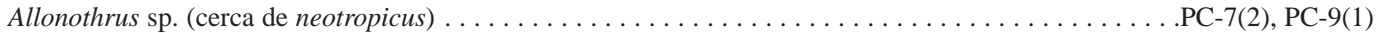

Archegozetes longisetosus Aoki, 1965 . . . . . . . . . . . . . . . . . . PC-3(7), PC-9(2), PC-12(2), PC-16(1)

NANHERMANNIIDAE

Cyrthermannia guadeloupensis Mahunka, $1985 \ldots \ldots \ldots \ldots \ldots \ldots \ldots \ldots \ldots \ldots \ldots \ldots$. . . . . . . . . . . . . . . . . . . . . . . . . . . . . . . . . . . .

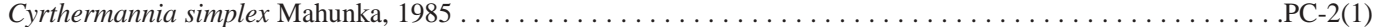

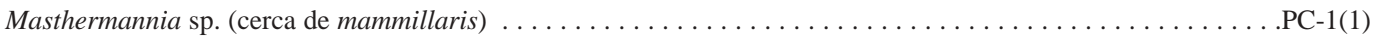

HERMANNIELLIDAE

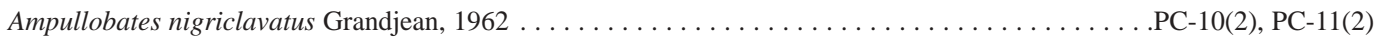

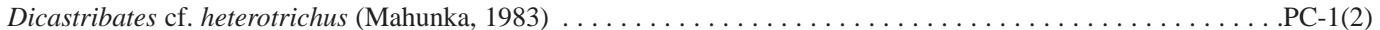

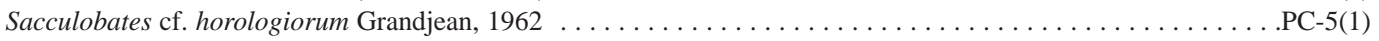

LIODIDAE

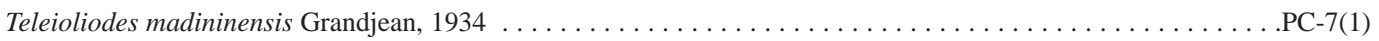

Teleioliodes cf. madininensis Grandjean, $1934 \ldots \ldots \ldots \ldots \ldots \ldots \ldots \ldots \ldots \ldots$. . . . . . . . . PC-2(1), PC-6(8), PC-12(1)

Teleioliodes zikani (Sellnick, 1930) . . . . . . . . . . . . . . . . PC-5(1), PC-6(8), PC-7(2), PC-10(3), PC-13(4)

PLATEREMAEIDAE

Plateremaeus berlesei Balogh y Mahunka, $1978 \ldots \ldots \ldots \ldots \ldots \ldots \ldots \ldots \ldots \ldots$. . . . . . . . . . . PC-4(1), PC-10(1)

MICROZETIDAE

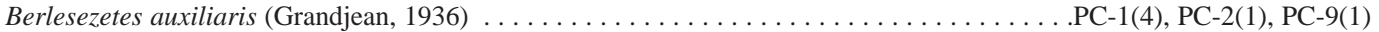

BASILOBELBIDAE

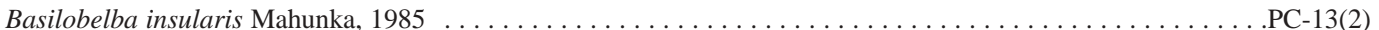

DAMAEOLIDAE

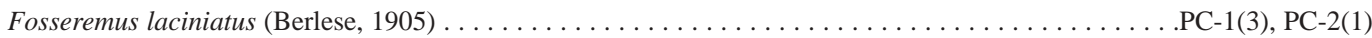

EREMOBELBIDAE

Eremobelba piffli Mahunka, $1985 \ldots \ldots \ldots \ldots \ldots \ldots \ldots \ldots \ldots \ldots \ldots \ldots \ldots \ldots$

EREMULIDAE

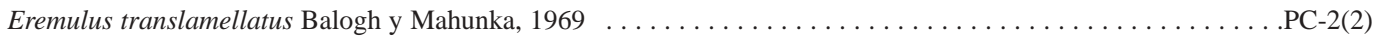

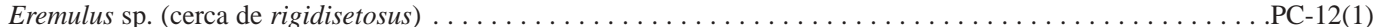

HETEROBELBIDAE

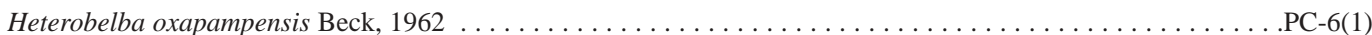

PELOPPIIDAE

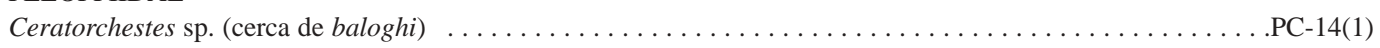

OTOCEPHEIDAE

Cavernocepheus sp. (cerca de monstruosus) . . . . . . . . . . . . . . . . . . . . . . . . . . . . 2(3)

Dolicheremaeus amazonicus Balogh y Mahunka, 1969 . . . . . . . . . . PC-6(4), PC-8(1), PC-10(1), PC-11(1), PC-12(1)

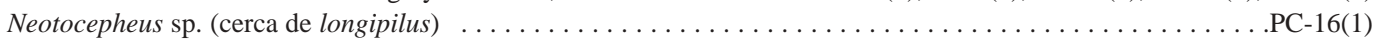

Pseudotocepheus cf. septemtuberculatus Balogh y Mahunka, $1978 \ldots \ldots \ldots \ldots \ldots \ldots$. . . . . . . . . . . . . . . . 1)

DAMPFIELLIDAE

Beckiella cf. foveolata Balogh y Mahunka, 1969

PC-8(1), PC-10(1), PC-13(1), PC-14(1) 
CARABODIDAE

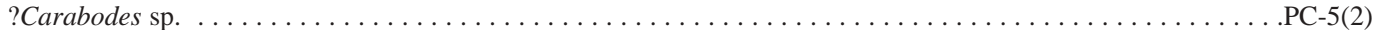

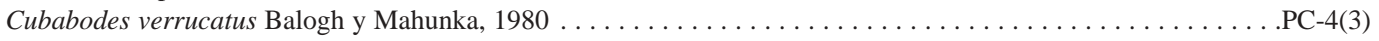

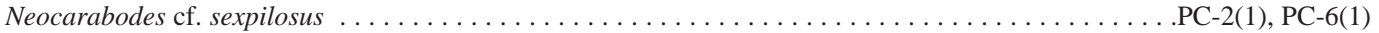

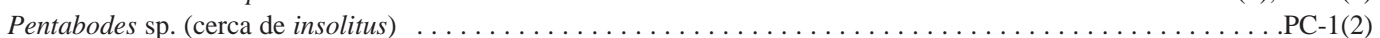

Yoshiobodes irmayi (Balogh y Mahunka, 1969) . . . . . . . . . . . . . . . . . . . . . . .PC-10(1), PC-12(1)

STERNOPPIIDAE

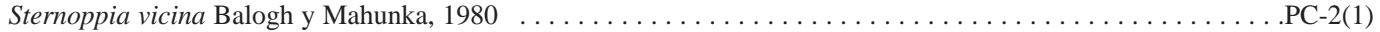

MACHUELLIDAE

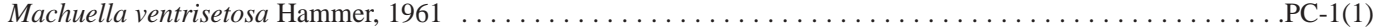

OPPIIDAE

OXYOPPIINAE

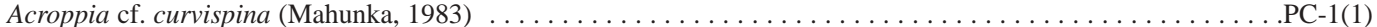

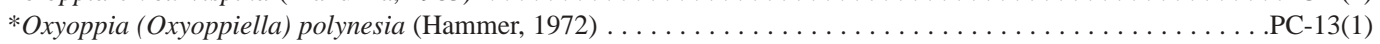

MYSTROPPIINAE

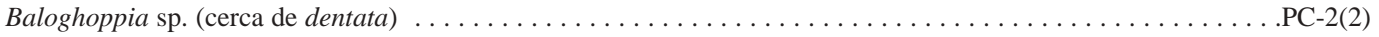

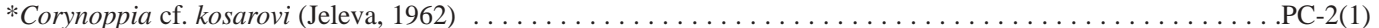

Striatoppia opuntiseta Balogh y Mahunka, $1968 \ldots \ldots \ldots \ldots \ldots \ldots \ldots \ldots \ldots \ldots \ldots \ldots$. . . . . . . . . . . . . . .

BRACHIOPPIINAE

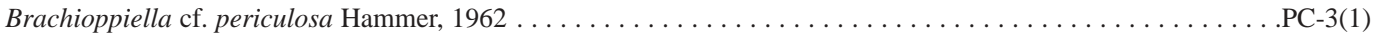

Gittella cf. maxima (Balogh y Mahunka, 1981)

PC-6(1), PC-15(1)

PULCHROPPIINAE

Varioppia cf. radiata Mahunka, $1985 \ldots \ldots \ldots \ldots \ldots \ldots \ldots \ldots \ldots \ldots \ldots \ldots$. . . . . . . . . . . . . . . . . . . . . . . . . . . . . . . . . . . PC-12(2), PC-14(1)

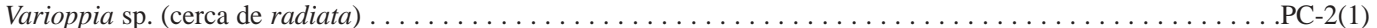

ARCOPPIINAE

Arcoppia cf. tripartita (Hammer, 1961)

MEDIOPPIINAE

*Discoppia (Cylindroppia) cylindrica (Pérez-Íñigo, 1965)

PC-13(1)

OPPIELLINAE

Oppiella nova (Oudemans, 1902)

OPPIINAE

Aeroppia mariehammerae n. nom. ..... . . . PC-2(2), PC-3(2), PC-7(1), PC-9(2), (para ?Aeroppia sp. de Hammer, 1962) PC-10(1), PC-12(11), PC-15(1), PC-16(2)

Neoamerioppia (Amerigloboppia) salvadoriensis (Woas, 1986) . . . . . . . . . . . . . . . . . . . . PC-11(1)

MULTIOPPIINAE

Pseudoamerioppia barrancensis (Hammer, 1961) . . . . . . . . . . . . . . . . . . . .PC-4(7), PC-8(3), PC-10(1)

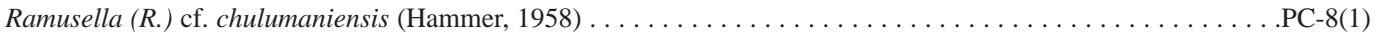

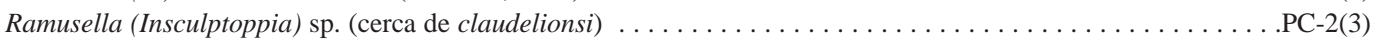

OXYAMERIDE

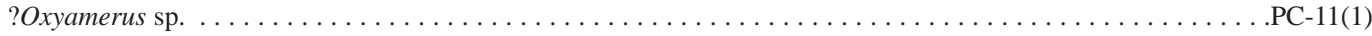

RHYNCHORIBATIDAE

Rhynchoribates edentatus Balogh y Mahunka, $1969 \ldots \ldots \ldots \ldots \ldots \ldots \ldots \ldots \ldots$. . . . . . . . . . PC-6(2), PC-10(1)

Rhynchoribates cf. spathulatus Balogh y Mahunka, $1969 \ldots \ldots \ldots \ldots \ldots \ldots \ldots \ldots \ldots \ldots$. . . . . . . . . . . . 
SUCTOBELBIDAE

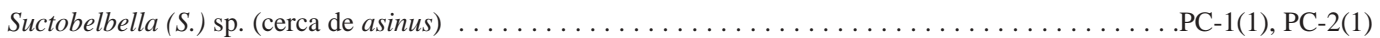

Suctobelbella (Discosuctobelba) complexa (Hammer, 1958) . . . . . . . . . . . . . . . . . PC-1(3), PC-2(1)

Suctobelbella (Discosuctobelba) cf. longiclava (Hammer, 1958) . . . . . . . . . . . . . . . . . . . PC-1(1)

Suctobelbella (Discosuctobelba) cf. subcomplexa (Bal. y Mah., 1968) . . . . . . . . . . . . . . PC-1(1), PC-10(1)

*Suctobelbella (Flagrosuctobelba) penicillata (Balogh y Mah., 1966) . . . . . . . . . . . . PC-1(3), PC-13(1), PC-14(1)

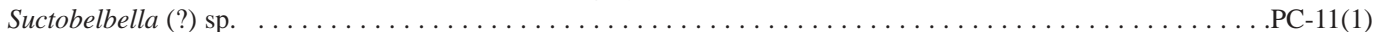

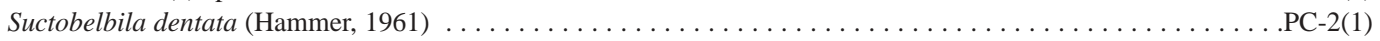

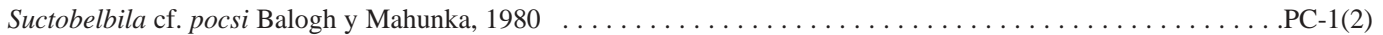

CERATOZETIDAE

Allozetes sp. (cerca de translamellatus) . . . . . . . . . . . . . . . . . . . . . . . . . . . . . . . . . .PC-1(14)

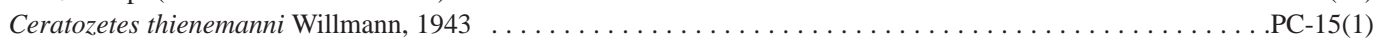

?Ceratozetes sp. . . . . . . . . . . . . . . . . . .

ORIBATELLIDAE

Lamellobates gyoergyi Balogh y Mahunka, $1977 \ldots \ldots \ldots \ldots \ldots \ldots \ldots \ldots \ldots$. . . . . . . 1(2), PC-2(1), PC-6(3), PC-10(1) Lamellobates molecula (Berlese, 1916) . . . . . . . . . . . . .PC-3(6), PC-5(1), PC-6(1), PC-7(3), PC-9(1), PC-13(2)

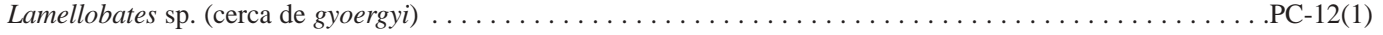

Oribatella sp. (cerca de serrula) . . . . . . . . . . . . . . . . . . . PC-7(3), PC-10(2), PC-14(3), PC-15(1)

GALUMNATIDAE

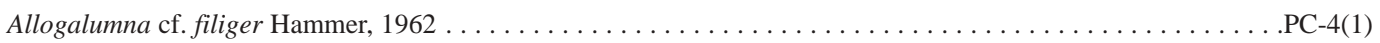

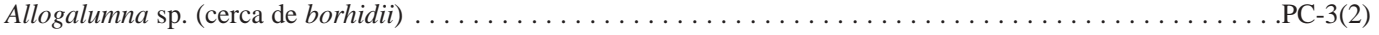

Galumna cf. angularis Jeleva, Scull y Cruz, $1984 \ldots \ldots \ldots \ldots \ldots \ldots \ldots \ldots \ldots \ldots \ldots \ldots \ldots$. . . . . . . . . . . . . . . PC-13(1)

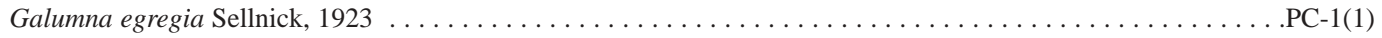

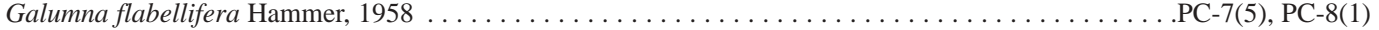

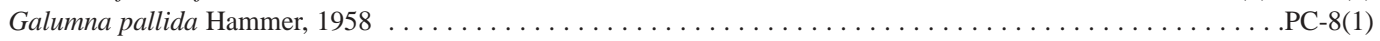

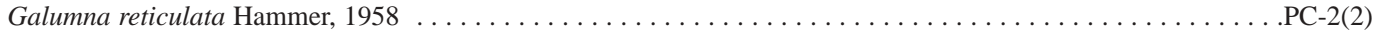

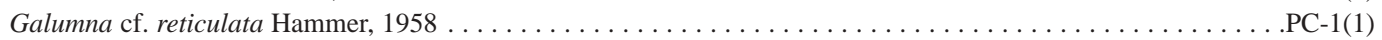

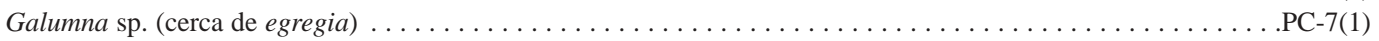

Galumna sp. (cerca de flabellifera $). \ldots \ldots \ldots \ldots \ldots \ldots \ldots \ldots \ldots \ldots \ldots \ldots$. . . . . . . . . . . PC-6(7), PC-12(2), PC-16(8)

Galumna sp. (cerca de pallida) . . . . . . . . . . . . . . . . . . . . . . . . . . PC-6(2), PC-7(1), PC-9(1)

Pergalumna cf. plumata Pérez-Íñigo y Baggio, 1986 . . . . . . . . . . .PC-1(7), PC-4(1), PC-6(3), PC-10(1), PC-12(9),

PC-14(1), PC-15(2)

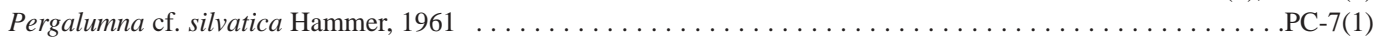

Pergalumna sp. (cerca de decorata) . . . . . . . . . . . . . . . . .PC-2(1), PC-3(1), PC-9(1), PC-10(1), PC-12(3)

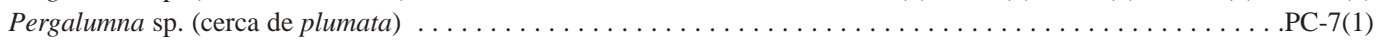

GALUMNELLIDAE

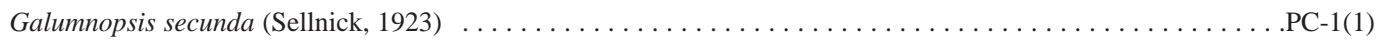

PARAKALUMMIDAE

Neoribates sp. (cerca de foraminiferus) …....PC-8(1), PC-9(1), PC-10(1), PC-12(18), PC-14(4), PC-15(2), PC-16(6)

MOCHLOZETIDAE

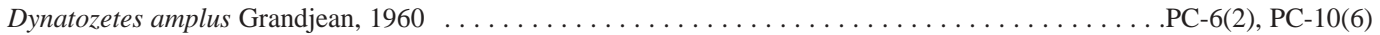

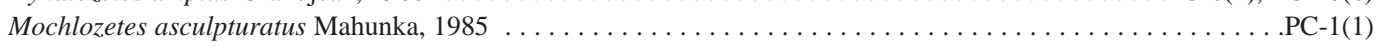

Mochlozetes penetrabilis Grandjean, 1930 . . . . . . . . . . . . . . . . . . . . . . PC-8(1), PC-9(1)

HAPLOZETIDAE

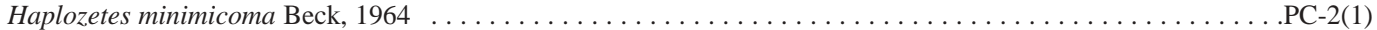

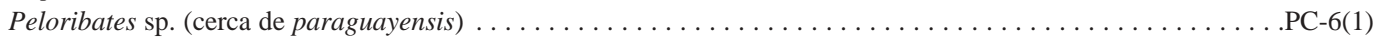

Rostrozetes ovulum (Berlese, 1908) . . . . . . . . . . . . . . . . . . . . . . . PC-2(4), PC-12(5), PC-16(2)

\section{XYLOBATIDAE}

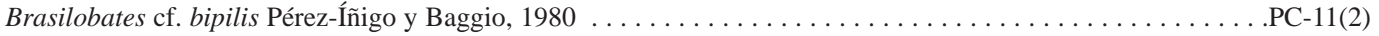

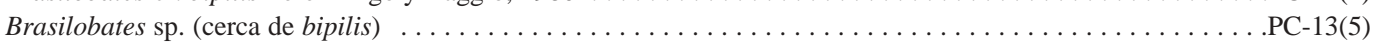




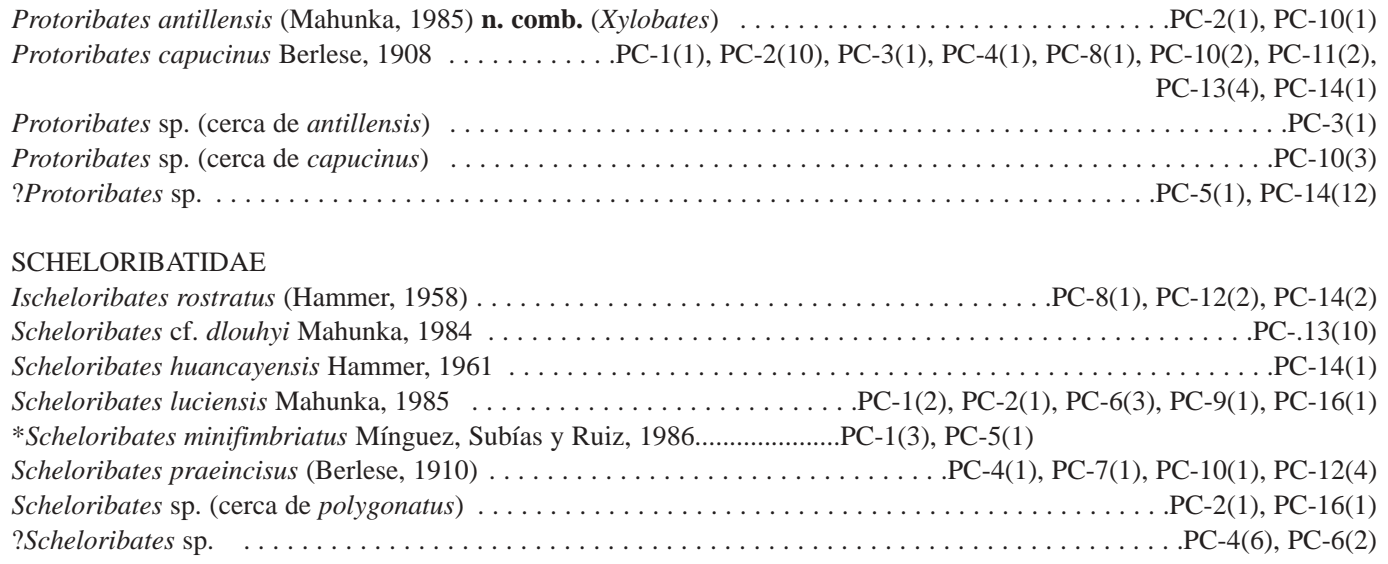

\section{DISCUSIÓN}

Teniendo en cuenta el tipo de muestreo realizado, muy puntual y específico, la cifra de 1053 ejemplares recolectados pertenecientes a 134 especies diferentes, suponen una contribución importante frente a las cerca de 2000 especies conocidas (entre descritas y citadas) en la región neotropical. Por lo que se refiere a Panamá en particular y Centroamérica en general, los datos aportados amplían y actualizan significativamente los escasos datos previos.

Con estas nuevas aportaciones, se incrementan el número de especies neotropicales conocidas, ya que se citan por vez primera las especies Gehypochthonius rhadamanthus Jacot, 1936; Epilohmannoides terrae Jacot, 1936; Rhysotritia hyeroglyphica (Berlese, 1916); Oxyoppia (Oxyoppiella) polynesia (Hammer, 1972); Corynoppia cf. kosarovi (Jeleva, 1962); Discoppia (Cylindroppia) cylindrica (Pérez-Íñigo, 1965); Suctobelbella (Flagrosuctobelba) penicillata (Balogh y Mahunka, 1966) y Scheloribates minifimbriatus Mínguez, Subías y Ruiz, 1986.

Desde el punto de vista nomenclatorial se crea la nueva combinación Protoribates antillensis para Xylobates antillensis Mahunka, 1985 y se da el nuevo nombre de Aeroppia mariehammerae a ?Aeroppia sp. Hammer, 1962, especie que dicha autora describe de los Andes chilenos y acompaña de un dibujo (Fig. 2), el cual es lo suficientemente explícito como para poder separar esta especie de todas las demás incluidas en el género, fundamentalmente por la configuración de las setas $p l$ y $h l$ y la longitud de las restantes setas notogastrales.

Las especies más frecuentes en las muestras estudiadas han sido Eohypochthonius gracilis (Jacot, 1936), Mesoplophora cubana Calugar y Vasiliu, 1977, Epilohmannia pallida Wallwork, 1962, Indotritia krakatauensis (Sellnick, 1923), Aeroppia mariehammerae $\mathbf{n}$. nom., Lamellobates molecula (Berlese, 1916), Pergalumna cf. plumata Pérez-Íñigo y Baggio, 1986, Protoribates capucinus Berlese, 1908 у Hemileius cf. microclava (Hammer, 1961), siendo esta última, la más abundante de todas con 217 individuos.

P. capucinus es la más cosmopolita de todas, apareciendo en otras regiones además de las tropicales; mientras que E. gracilis, E. pallida y L. molecula son especies circuntropicales.

\section{AGRADECIMIENTOS}

Expresamos nuestra gratitud a la Agencia Española de Cooperación Internacional en 


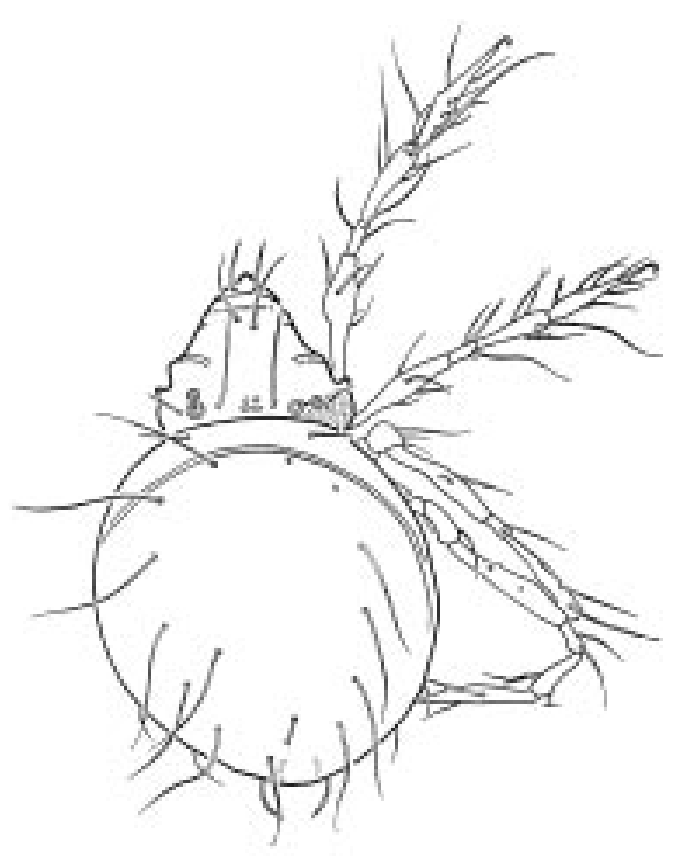

Fig. 2. Vista dorsal de Aeroppia mariehammerae n. nom. según Hammer, 1962.

Fig. 2. Dorsal view of Aeroppia mariehammerae n. nom. according to Hammer, 1962.

Panamá, tanto por su apoyo en Panamá, como por su ayuda logística en los viajes y estancia en la Estación Biológica de la isla de Coiba; agradecimiento que hacemos extensivo a Santiago Castroviejo, promotor y coordinador del proyecto científico de inventario del Parque Nacional. También deseamos agradecer a los efectivos de INRENARE y a los miembros de la policía de Panamá, destacados en la Estación Biológica, por su apoyo en los movimientos y trabajo de campo en la isla. Muy especialmente damos las gracias a Alicia Ibáñez por su compañía, aliento y ayuda prestada de muy variados modos durante nuestra estancia en la Estación Biológica y en los muestreos, así como también a Antonio Arillo. El trabajo ha sido parcialmente subvencionado por la Agencia española de Cooperación Internacional en Panamá y por el proyecto DGES PB97-1241.

\section{RESUMEN}

Dentro del programa para el inventario de la fauna y flora del Parque Nacional de la Isla de Coiba (Panamá), se presenta un primer listado de los ácaros oribátidos (Acari: Oribatei) que habitan en el parque. El material objeto de este estudio, procede de diversos muestreos edáficos, incluyendo hojarasca y humus, realizados en el sitio en julio de 1998. Para un total de 1053 ejemplares colectados en las muestras se han identificado 134 especies diferentes, de las cuales, practicamente todas son nuevas para Panamá y 8 especies se citan por vez primera en la región neotropical. Se da un nuevo nombre: Aeroppia mariehammerae n.nom. para Aeroppia sp. (Hammer, 1962) y una combinación nueva para Protoribates antillensis (Mahunka, 1985) n. comb. (Xylobates).

\section{REFERENCIAS}

Balogh, J. \& P. Balogh. 1988. The soil mites of the world 2. Oribatid mites of the neotropical region I. Elsevier, Amsterdam. 335 p.

Balogh, J. \& P. Balogh. 1990. The soil mites of the world 3. Oribatid mites of the neotropical region II. Elsevier, Amsterdam. 333 p.

Berlese, A. 1888. Acari Austro-Americani quos collegit Alysius Balzan. Bull. Soc. Ent. Ital. 20: 215-222.

Camacho, A.I., E. Bello \& A. García-Valdecasas. 1997. Los invertebrados de agua dulce de la isla de Coiba (Panamá), pp. 127-153. In Flora y Fauna del Parque Nacional de Coiba (Panamá). S. Castroviejo (ed.). AECI. Madrid.

Castroviejo, S. (ed.) 1997. Flora y Fauna del Parque Nacional de Coiba (Panamá) Inventario preliminar. AECI. Madrid. 534 p.

Coiffait, H. 1958. Contribution à la connaissance des Coléoptères du sol. Thèse Doctoral. Univ. Touluse, Vie et Mileu 7: 1-204.

Ghilarov, M.S. \& D.A. Krivolutsky. 1975. Claves de identificación de ácaros edáficos Sarcopteriformes (en ruso). Nauka, Moscú. 492 p.

Hammer, M. 1958. Investigations on the Oribatid fauna of the Andes Mountains. I. The Argentine and Bolivia. Biol. Skr. Dansk. Vid. Selsk X (1): 1-129.

Hammer, M. 1962. Investigations on the Oribatid fauna of the Andes Mountains. III. Chile. Biol. Skr. Dansk. Vid. Selsk XIII (2): 1-96.

Subías, L.S. \& J. Gil-Martín. 1997. Systematic and biogeographic checklist on Oribatids from Western Mediterranean (Acari, Oribatida). Ann. Mus. Civ. Stor. Nat. "G. Doria" 91: 459-498. 
